\title{
In an open publishing house not so far, far away...
}

Kenneth R. Kaufman, Gin S. Malhi, Amanda Baxter and Kamaldeep Bhui

\section{Summary}

As BJPsych Open completes its first circle around the sun and marks its first anniversary, we share with you its strengths and advantages that underpin its success as a new journal. First and foremost, the editorial team has maintained rigorous scientific standards while pursuing an open access publishing model that, by design, accommodates a broad range of clinical and scientific topics. Fundamental to BJPsych Open's mission has been our policy of accepting papers that are both methodologically sound and intellectually stimulating. The calibre of the journal has already been recognised, with recent notification of indexing all its content in PubMed Central. This reflects the quality of submissions and is the result of concerted efforts by the authors, the editorial board, the many selfless reviewers and our dedicated staff in the journal office. We urge you to join us on this exciting journey and look to your input as authors, readers and reviewers to help shape this fledgling enterprise, destined to become a force to be reckoned with.

\section{Declaration of interests \\ None.}

\section{Copyright and usage}

(c) The Royal College of Psychiatrists 2016. This is an open access article distributed under the terms of the Creative Commons NonCommercial, No Derivatives (CC BY-NC-ND) license.
Kenneth R. Kaufman (pictured) is Professor of Psychiatry, Neurology and Anesthesiology at Rutgers Robert Wood Johnson Medical School (New Brunswick) and Deputy Editor of BJPSych Open. Gin S. Malhi is Chair of Psychiatry at the University of Sydney, Deputy Editor of BJPsych Open and Editor of the Australian and New Zealand Journal of Psychiatry. Amanda Baxter co-leads the Policy and Research Translation Group at the Queensland Centre for Mental Health Research, and is Associate Editor of BJPsych Open. Kamaldeep Bhui is Professor of Cultural Psychiatry and Epidemiology at Queen Mary University of London, an Honorary Consultant Psychiatrist in the Eas London NHS Foundation Trust and head of department at Barts and The London School of Medicine and Dentistry. He is Editor of the British Journal of Psychiatry and BJPsych Open as well as of the International Journal of Culture and Mental Health

\section{Our first chapter}

The genesis and early stages of a new peer-review academic journal are expected to cause labour pains and be affected by periodic developmental delays, but perhaps BJPsych Open is an exception. Within the first 12 months since its launch, BJPsych Open has published 70 articles consisting of 64 papers, reviews and short reports (423 pages) and 6 editorials (16 pages). In addition, the journal has achieved PubMed Central indexing, which speaks directly to the quality of the articles accepted following rigorous scientific review and indirectly, but equally importantly, to the Herculean efforts of the reviewers, editorial board and journal office.

BJPsych Open was initially envisioned as a potential cascade journal from the British Journal of Psychiatry to ensure quality papers being published online as open access papers (free and easy availability for all readers) for the benefit of academics, clinical researchers, clinicians, and, last but not least, members of the Royal College of Psychiatrists. This was felt necessary for, with limited print space in the British Journal of Psychiatry, cascading articles to BJPsych Open, with authors' consent, permitted excellent articles to be redirected and published after further rigorous review. BJPsych Open as an online open access journal has helped to address a key College goal - optimising dissemination of research and critical ideas pertinent to mental health with an increased international presence. BJPsych Open has blossomed into an independent journal that now serves as a cascade journal only in part - and instead, receives a substantial number of excellent submissions in its own right. Part of its success is because it functions mainly as a broad-based general psychiatry journal that aims to accommodate papers addressing a diverse range of clinical and research topics, spanning child and adolescent psychiatry, adult, consultation-liaison, neuropsychiatric, forensic and geriatric populations. Truly, both College members and non-members from all areas of psychiatry and affiliated fields will always find an edifying paper suited to their specialisation.

Fundamental to BJPsych Open is that each paper, whether an editorial, research article, review or short report, must be both methodologically sound and intellectually stimulating, with meaningful insights and relevance to the field. Speaking to these points, the first year of BJPsych Open saw papers ranging from epidemiological to fMRI studies, from ethics to historical suicide rates following The Great War, from an adoptee and refugee comparative study to the impact of peacekeeping on mental health, from translational research to well-controlled randomised psychological and pharmaceutical trials, from theory to metaanalyses, from personality disorders to the psychological impact of natural disasters and poverty, from risk factor analyses to a cutting-edge editorial on ketamine.

We can publish articles with considerable flexibility with respect to format and length and endeavour to publish all papers within 28 days of acceptance, offering authors an excellent service, but also ensuring the timely democratisation of knowledge through public access.

Without authors and article submissions there would be no academic journals. Thus, we have been very fortunate to have received 250 submissions from 1457 authors representing 38 countries, of which 72 papers have been accepted. The process of submission to acceptance has involved the efforts of a dedicated editorial board which will be expanded as the journal continues to grow. The success of BJPsych Open has been based on the willingness of the authors and handling editors to have as many revisions as required to optimise the quality of published papers. Further, BJPsych Open is grateful to a very dedicated editorial and production staff. Most importantly, behind every successful journal are the truly unsung heroes, the conscientious reviewers 
(318 from 39 countries) without whom no article would see the light of day. Finally, we thank our readers from more than 70 countries and 6 continents.

\section{Looking forward to the next chapters}

As we mark BJPsych Open's first birthday, we remain thankful to all those who have made this journey and the journal possible. Without the vision and support of the College, BJPsych Open would not exist. Now, as we embark on our 'second chapter', we focus on maintaining the momentum from this first year with the expectation of achieving continued success. To accomplish this, we look to your input for further guidance and remain always responsive to both the College and all the readers of BJPsych Open.
Kenneth R. Kaufman, MD, FRCPsych, Rutgers Robert Wood Johnson Medical School, New Brunswick, New Jersey, USA; Gin S. Malhi, MD, FRCPsych, University of Sydney, Sydney, Australia; Amanda Baxter, PhD, Queensland Centre for Mental Health Research, Brisbane, Queensland, Australia; Kamaldeep Bhui, MD, FRCPsych, Queen Mary University of London, London, UK.

Correspondence: Kenneth R. Kaufman, Departments of Psychiatry, Neurology and Anesthesiology, Rutgers Robert Wood Johnson Medical School, 125 Paterson Street, Suite \#2200, New Brunswick, NJ 08901, USA. Email: kaufmakr@rwjms.rutgers.edu

First received 20 Jun 2016, final revision 13 Jul 2016, accepted 14 Jul 2016 